\title{
Lipid composition and dynamics of cell membranes of Bacillus stearothermophilus adapted to amiodarone
}

\author{
Sónia M.L.J. Rosa a, Maria do Carmo Antunes-Madeira b, Manuel J. Matos a, \\ Amália S. Jurado ${ }^{a}$, Vítor M.C. Madeira ${ }^{\mathrm{a}, *}$ \\ a Centro de Neurociencias, Departamento de Bioquímica, Universidade de Coimbra, Apartado 3126, 3001-401 Coimbra, Portugal \\ b Centro de Neurociências, Departamento de Zoologia, Universidade de Coimbra, 3004-517 Coimbra, Portugal
}

Received 26 April 2000; received in revised form 14 August 2000; accepted 15 August 2000

\begin{abstract}
Bacillus stearothermophilus, a useful model to evaluate membrane interactions of lipophilic drugs, adapts to the presence of amiodarone in the growth medium. Drug concentrations in the range of 1-2 $\mu \mathrm{M}$ depress growth and $3 \mu \mathrm{M}$ completely suppresses growth. Adaptation to the presence of amiodarone is reflected in lipid composition changes either in the phospholipid classes or in the acyl chain moieties. Significant changes are observed at $2 \mu \mathrm{M}$ and expressed by a decrease of phosphatidylethanolamine (relative decrease of 23.3\%) and phosphatidylglycerol $(17.9 \%)$ and by the increase of phosphoglycolipid (162\%). The changes in phospholipid acyl chains are expressed by a decrease of straight-chain saturated fatty acids (relative decrease of $12.2 \%$ ) and anteiso-acids (22\%) with a parallel increase of the iso-acids (9.8\%). Consequently, the ratio straight-chain/branched iso-chain fatty acids decreases from 0.38 (control cultures) to 0.30 (cultures adapted to $2 \mu \mathrm{M}$ amiodarone). The physical consequences of the lipid composition changes induced by the drug were studied by fluorescence polarization of diphenylhexatriene and diphenylhexatriene-propionic acid, and by differential scanning calorimetry. The thermotropic profiles of polar lipid dispersions of amiodarone-adapted cells are more similar to control cultures (without amiodarone) than those resulting from a direct interaction of the drug with lipids, i.e., when amiodarone was added directly to liposome suspensions. It is suggested that lipid composition changes promoted by amiodarone occur as adaptations to drug tolerance, providing the membrane with physico-chemical properties compatible with membrane function, counteracting the effects of the drug. (C) 2000 Elsevier Science B.V. All rights reserved.
\end{abstract}

Keywords: Amiodarone; Phospholipid composition; Fatty acid composition; Lipid dynamics; Bacillus stearothermophilus

\section{Introduction}

Amiodarone (2-butyl-3-(3',5'-diiodo-4' $\alpha$-diethylaminoethoxybenzoyl)-benzofuran; AMD, Fig. 1), widely used as an antiarrhythmic, is traditionally de-

\footnotetext{
* Corresponding author. Fax: +351-239-826798;

E-mail: vmcm@ci.uc.pt
}

scribed as a class III drug [1] due to its ability to prolong cardiac action potential duration, with consequent slow-down of cardiac rhythm [2]. Unfortunately, the benefits of its use are accompanied by a number of side effects on lungs, liver, nervous system, thyroid gland, cornea and skin [2], which may limit its use.

In spite of the efforts in recent years, the molecular mechanisms underlying AMD antiarrhythmic action and undesirable effects still escape coherent scientific 


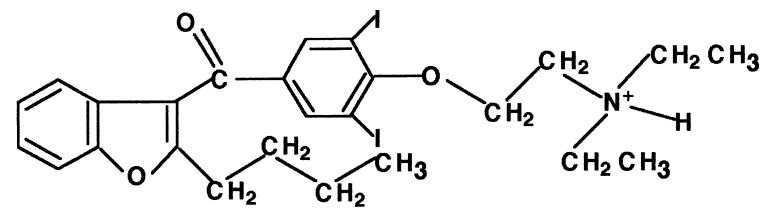

Fig. 1. Structure of amiodarone.

understanding. Therefore, identification of the molecular mechanisms of action is of obvious interest in the development of analogues with improved biological selectivity.

The amphiphilic character of AMD and its low water solubility, $0.72 \mathrm{mg} / \mathrm{ml}$ [3], are signs of a high affinity of AMD for membrane lipids. Actually, the partition coefficient of AMD into membranes is above 10000 [4]. Therefore, a significant therapeutic activity of the drug and side effects may relate with the interaction of AMD with membrane components.

Several reports confirm that AMD affects membrane function [5-11]. Effects at this level might result from changes induced by the drug on the bilayer physico-chemical properties, since these are crucial to membrane function $[12,13]$. Consequently, the electrophysiologic effects of AMD and most of its side effects appear to be membrane-connected, caused by changes in membrane lipid organization and dynamics. Indeed, AMD affects the lipid physical state and organization, either in models or in native membranes [14-18]. However, all these effects require further detailed clarification.

The intensive studies undertaken in the past two decades revealed complex biochemical actions in the complex potential membrane target sites. Interpretation of data is, therefore, difficult owing to putative multiple interactions with basic membrane mechanisms of eukaryotic cells.

Bacterial cells are often good tools for modeling the action of membrane active drugs [19-21]. Most of the bacterial vital cell functions are ascribed to the plasma membrane [22-25] and, consequently, perturbations of membrane structure and dynamics will impair viability and growth, making it possible to correlate the effects at the molecular level with physiological events. Furthermore, results concerning drug effects on the biophysical properties of membrane lipids of prokaryotes and their repercussions on membrane function can be extrapolated to eu- karyotes [12,19-21]. Moreover, bacteria are very sensitive to adverse agents which affect the physical state of membrane lipids, promoting alterations of membrane lipid composition [21,26-28]. Thus, the efficient molecular mechanisms of adaptation may contribute to clarifying the molecular mechanisms of action of adverse agents. The eubacterium Bacillus stearothermophilus has been extensively used in our laboratory for this type of study [20,21,27,28]. Furthermore, it has also been used as a model to study AMD-membrane interactions. The tolerance of B. stearothermophilus to AMD [29] may be related to changes in lipid composition and, consequently, to changes in the organization and dynamics of membrane lipids. Therefore, the purpose of this work is to evaluate putative alterations of lipid composition in response to the stress imposed by AMD and the consequent alterations on membrane physical properties. These studies are relevant to elucidate the physico-chemical basis of AMD interactions with biomembranes.

\section{Materials and methods}

\subsection{Chemicals}

Amiodarone was provided by Sanofi-Labaz Research, Inc., Montpellier, France. The probes DPH (1,6-diphenyl-1,3,5-hexatriene) and DPH-PA (3-( $p$ (6-phenyl)-1,3,5-hexatrienyl) phenylpropionic acid) were purchased from Molecular Probes, Inc., Eugene, OR, USA.

\subsection{Growth conditions}

The strain of B. stearothermophilus used was supplied by Mast Laboratories, UK (lot \#7953). The conditions of its maintenance and growth have been previously described [27]. Liquid cultures were started with an early stationary phase inoculum from a complex medium (L-broth) and were grown in 11 Erlenmeyer flasks containing $200 \mathrm{ml}$ of growth medium. The flasks were shaken at $130 \mathrm{rpm}$ in a New Brunswick water bath shaker, at $65^{\circ} \mathrm{C}$. AMD was added to the growth medium from a concentrated ethanolic solution in order to obtain concentrations ranging from 1 to $3 \mu \mathrm{M}$. Growth was monitored by 
measuring the turbidity at $610 \mathrm{~nm}$ in a Bausch and Lomb Spectronic 21 spectrophotometer.

\subsection{Extraction of polar lipids}

Growth was stopped at the end of the exponential phase, before the beginning of the stationary phase, by cooling the cultures on ice. Bacterial cells were harvested by centrifugation and washed three times with buffer (10 mM Tris- $\mathrm{HCl}, \mathrm{pH} 7.0)$ and resuspended in the same buffer supplemented with $15 \mathrm{mM} \mathrm{MgCl}$. Cells were converted to protoplasts under the action of $0.2 \%(\mathrm{w} / \mathrm{v})$ lysozyme, for $30 \mathrm{~min}$, at $37^{\circ} \mathrm{C}$. Lipids were extracted by the method of Bligh and Dyer [30] and quantified by measuring the amount of inorganic phosphorus [31], after hydrolysis of the extracts in $70 \% \mathrm{HClO}_{4}$, at $180^{\circ} \mathrm{C}$, for $60 \mathrm{~min}$ [32]. The polar lipids were purified by preparative thin layer chromatography (TLC) on $2 \mathrm{~mm}$ thick silica gel plates (Merck, Darmstadt, Germany), developed in acetone to wash nonpolar components. The polar lipids remaining in the application zone were extracted with a mixture of $\mathrm{CHCl}_{3}$ : $\mathrm{CH}_{3} \mathrm{OH}: \mathrm{H}_{2} \mathrm{O}(45: 45: 10$, v/v), followed by phase separation. The polar lipid phase was collected and evaporated to dryness in a rotary evaporator. The lipid residue was dissolved in chloroform and stored under nitrogen atmosphere, at $-20^{\circ} \mathrm{C}$.

\subsection{Phospholipid analysis}

The polar lipid classes were separated by TLC on plates of silica gel $\mathrm{H}$ (0.5 mm thickness), using for development the solvent mixture $\mathrm{CHCl}_{3}$ : $\mathrm{CH}_{3} \mathrm{OH}: \mathrm{H}_{2} \mathrm{O}(65: 25: 4$, v/v). Separated lipid classes were visualized by iodine vapor reaction [33] or with specific spray reagents: molybdate reagent for lipids containing phosphate [34], ninhydrin reagent for free amino groups [33] and $\alpha$-naphthol reagent for carbohydrates [35]. Identification of lipid classes was performed with reference to standards run along with the samples on the TLC plates. The phospholipid bands were scraped from the plates and quantified by standard procedures [31,32].

\subsection{Fatty acid analysis}

The fatty acid composition of bacterial polar lipids was determined as previously described [36] with some modifications. A lipid sample (ca. $1 \mu \mathrm{mol}$ ) was transferred into a transesterification tube and the solvent was evaporated with an $\mathrm{N}_{2}$ stream. The dry residue was mixed with $5 \mathrm{ml}$ of methylation solution $\left(5 \% \mathrm{H}_{2} \mathrm{SO}_{4}\right.$ in $\mathrm{CH}_{3} \mathrm{OH}$, w/v) and saturated with $\mathrm{N}_{2}$. Transmethylation was carried out for $2 \mathrm{~h}$ at $70^{\circ} \mathrm{C}$, under $\mathrm{N}_{2}$ atmosphere, after tightly sealing the tube. Then, the tube was cooled and the fatty acid methyl esters were extracted with $10 \mathrm{ml}$ hexane and $5 \mathrm{ml}$ quartz distilled water. The mixture was vortexed for $30 \mathrm{~s}$ and then centrifuged for $2 \mathrm{~min}$ at $1000 \mathrm{rpm}$ to accelerate and improve phase separation. The upper phase (hexane) was collected and the lower phase was reextracted with hexane. Traces of water in the hexane extracts were removed by adding about $1 \mathrm{~g}$ of anhydrous sodium sulfate. The extracts were filtered, evaporated to dryness and then dissolved in about $200 \mu \mathrm{l}$ of $\mathrm{CS}_{2}$.

The fatty acid methyl esters were analyzed in a Varian gas-liquid chromatograph, series 1400, equipped with an open capillary column SGE 25QC5/BPX70 (25 m, $0.53 \mathrm{~mm})$ eluted with $\mathrm{He}$ at $3.0 \mathrm{ml} / \mathrm{min}$. A temperature program was started at $140^{\circ} \mathrm{C}$, held for $14 \mathrm{~min}$, and then increased to $210^{\circ} \mathrm{C}$ at $4^{\circ} \mathrm{C} / \mathrm{min}$, using $25 \mathrm{ml} \mathrm{He} / \mathrm{min}$ as make-up gas in the detector system. Peaks were identified by comparing retention times with those of authentic standards (fatty acid methyl esters obtained from Supelco and Alltech) and quantified by integration of peaks with the aid of a computerized integrator.

\subsection{Liposomes for fluorescence polarization studies}

Aliquots from bacterial polar lipids, in $\mathrm{CHCl}_{3}$, containing $1.34 \mathrm{mg}$ of lipid, were evaporated to dryness in a rotary evaporator. The resulting lipid film was then hydrated with $5 \mathrm{ml}$ of $50 \mathrm{mM} \mathrm{KCl}, 10 \mathrm{mM}$ Tris-maleate, $\mathrm{pH}$ 7.0, and dispersed, under $\mathrm{N}_{2}$ atmosphere, at $50^{\circ} \mathrm{C}$, by gentle shaking. The liposome suspension was then vortexed for $1 \mathrm{~min}$ and allowed to stabilize overnight.

\subsection{Incorporation of the probes and amiodarone into liposomes}

DPH and DPH-PA in dimethylformamide were injected (few $\mu$ l) into membrane suspensions 
(345 $\mu \mathrm{M}$ in total lipid) to give a final lipid/diphenylhexatriene probes molar ratio of about 300 . The mixture was initially vigorously vortexed for $10 \mathrm{~s}$ and then AMD was added from concentrated ethanolic solutions. It was ascertained that added concentrations of AMD were within the solubility range. The mixture was incubated, at $42^{\circ} \mathrm{C}$, in the dark to protect the probe, for a period of $18-20 \mathrm{~h}$ to reach equilibrium, since the drug has to penetrate multiple bilayers. Control samples received equivalent volumes of dimethylformamide and ethanol. Added solvent volumes, always very small (few $\mu \mathrm{l}$ ), had negligible effects on the measurements.

\subsection{Fluorescence polarization measurements}

Fluorescence polarizations were determined in a Perkin-Elmer spectrofluorometer, model MPF-3, equipped with a thermostated cell holder. The excitation was set at $360 \mathrm{~nm}$ and the emission at $450 \mathrm{~nm}$. The excitation and emission band widths were 6 and $8 \mathrm{~nm}$, respectively. The temperature of the sample was checked with an accuracy of $\pm 0.1^{\circ} \mathrm{C}$, using a thermistor thermometer. The degree of fluorescence polarization $(P)$ was calculated according to Shinitzky and Barenholz [37] from the equation:

$P=\frac{I_{\|}-I_{\perp} G}{I_{\|}+I_{\perp} G}$

where $I_{\|}$and $I_{\perp}$ are the intensities of the light emitted with its polarization plane parallel (॥) and perpendicular $(\perp)$ to that of the exciting beam. The grating correction factor for the optical system $(G)$ is given by the ratio of vertically to horizontally polarized emission components, when the excitation light is polarized in the horizontal plane [38]. Depolarization effects as a consequence of scattering were taken into account and included in the grating factor measured at each temperature. All the fluorescence measurements were corrected for the contribution of light scattering by using controls with membranes, but without added probes. It should be stated that AMD, at the concentrations used in the present work, has no effect on the fluorescence lifetime of the probes [6].

DPH is a lipophilic probe known to be localized in the hydrophobic core of the membrane and provides structural information in this region [37]. On the other hand, DPH-PA is anchored in close proximity to the bilayer surface by its propionate group and the DPH moiety is embedded in the phospholipid acyl chains. Thus, it probes the bilayer lipid environments close to the surface, i.e., in the outer bilayer regions [39]. A high degree of polarization reflects a limited rotational diffusion of the probes and, therefore, reflects a high structural order or low membrane fluidity and vice versa. The term fluidity is used here as being inversely proportional to the degree of fluorescence polarization of DPH and DPH-PA probes and essentially reflects the rate of motion of phospholipid acyl chains.

\subsection{Differential scanning calorimetry (DSC)}

For DSC measurements liposomes of polar lipid extracts were used, starting with ca. $640 \mu \mathrm{mol}$ of lipid extract. The lipid dispersions were allowed to equilibrate in the dark, for $18-20 \mathrm{~h}$, and then centrifuged at $450000 \times \mathrm{g}$, for $45 \mathrm{~min}$, at $4^{\circ} \mathrm{C}$. The wet pellets were hermetically sealed into aluminum pans and placed in a Perkin-Elmer Pyris 1 differential scanning calorimeter. The DSC measurements were performed using the software provided by Perkin-Elmer. To check the reproducibility of the results, three heating scans were recorded for each sample. Cooling scans yielded thermograms very similar to the heating scans, but and according to the literature [40], the transitions in cooling curves were shifted by about $1{ }^{\circ} \mathrm{C}$ to lower temperatures. Therefore, due to the supercooling phenomenon, accurate thermotropic transitions are evaluated from heating curves. Consequently, heating scans have been used in this work. The onset and completion of the phase transition were determined from the baseline of the thermograms [41].

At the end of the experiments, the aluminum pans were opened and the samples dissolved in a chloroform/methanol $(5: 1, \mathrm{v} / \mathrm{v})$ mixture. Phospholipids were quantitated by measuring inorganic phosphorus [31] released after hydrolysis of the extracts in $70 \%$ $\mathrm{HClO}_{4}$ at $180^{\circ} \mathrm{C}, 60 \mathrm{~min}$ [32].

\subsection{Analysis of data and statistical methods}

The values of phospholipid and fatty acid contents for control cultures and cultures grown in the pres- 


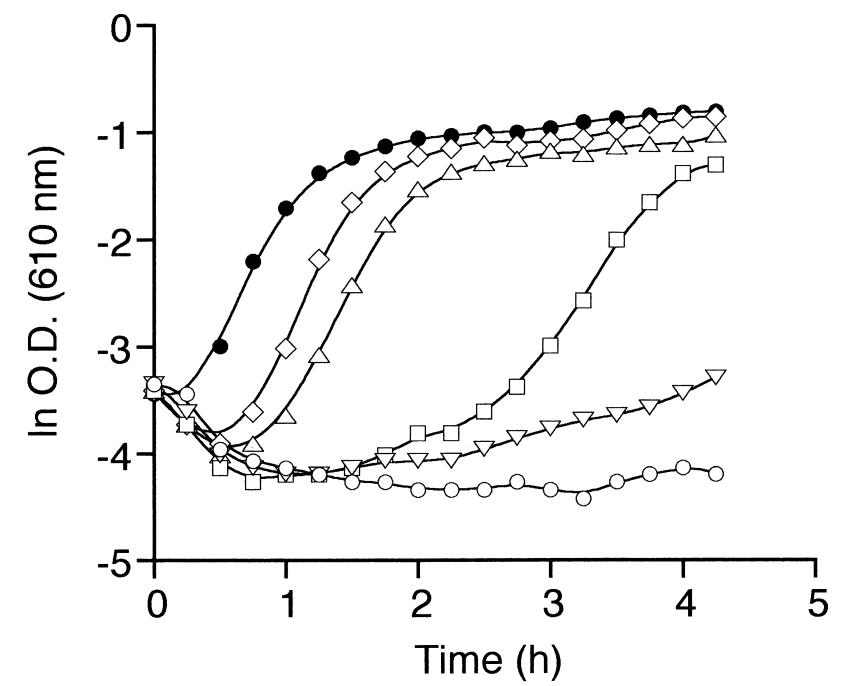

Fig. 2. Growth of cultures of B. stearothermophilus, at $65^{\circ} \mathrm{C}$, in a complex medium (L-broth) in the absence of amiodarone (control cultures, $\bullet$ ) and in the presence of increasing concentrations of the drug $(1.0 \mu \mathrm{M}, \diamond ; 1.5 \mu \mathrm{M}, \Delta ; 2.0 \mu \mathrm{M}, \square ; 2.5$ $\mu \mathrm{M}, \nabla$; and $3.0 \mu \mathrm{M}, \bigcirc)$. Growth was measured as absorbance at $610 \mathrm{~nm}$. The standard deviations of the experimental points were too small to be displayed by error bars.

ence of $1 \mu \mathrm{M}$ and $2 \mu \mathrm{M}$ AMD are means \pm S.D. of three independent experiments. Significance was calculated with Student's $t$-test of paired comparisons. This statistical analysis was also applied to the $T_{\mathrm{m}}$ temperatures of DSC heating scans.

\section{Results and discussion}

According to previous data [29], AMD added to the growth medium of $B$. stearothermophilus in a concentration range of $1-2.5 \mu \mathrm{M}$ induces progressive negative impact on bacterial growth. Thus, a progressive increase in the lag time, a decrease of the specific growth rate and a decrease of the final cell density are observed (Fig. 2). Furthermore, at a drug concentration of $3 \mu \mathrm{M}$, growth is completely inhibited. The efficient molecular mechanisms of adaptation of the bacterium to external agents $[21,28,42]$ indicate that the tolerance to AMD may relate to changes in lipid composition reflected in lipid packing and organization [43]. However, at a drug concentration of $3 \mu \mathrm{M}$, the bacterium was no longer able to compensate for the presence of the drug within the membrane. Therefore, the present work explores the lipid composition changes and the consequent biophysical modifications, when AMD $(1$ and $2 \mu \mathrm{M})$ is added to the bacterial growth medium.

\subsection{Lipid composition studies}

The membrane lipid composition of cultures of B. stearothermophilus grown in the absence (control cultures) and in the presence of 1 and $2 \mu \mathrm{M}$ AMD (adapted cultures) was examined. Lipid composition studies were performed for polar lipids, which account for $80-90 \%(\mathrm{w} / \mathrm{w})$ of the total lipid extract [42]. The remaining lipid components, the neutral lipids, accounting for about $10 \%$, were not studied. As listed in Table 1, and according to previous studies $[21,28,42]$ the polar lipid fraction contains phosphatidylethanolamine (PE), diphosphatidylglycerol (DPG), phosphatidylglycerol (PG) and a phosphoglycolipid (PGL). Trace amounts of two unidentified phospholipids $\left(\mathrm{X}_{1}\right.$ and $\left.\mathrm{X}_{2}\right)$ were also detected. The major class is $\mathrm{PE}$, which accounts for about $59 \mathrm{~mol} \%$ of the total phosphorus content. The other classes, i.e., DPG, PG and PGL, account for 26, 5.6 and $6.1 \mathrm{~mol} \%$, respectively. The minor phospholipids $\mathrm{X}_{1}$ and $\mathrm{X}_{2}$ account for 1.2 and $0.2 \mathrm{~mol} \%$, respectively. As also indicated in Table 1, cultures grown

Table 1

Phospholipid composition of B. stearothermophilus membranes, as affected by AMD

\begin{tabular}{llllllll}
\hline$[\mathrm{AMD}](\mu \mathrm{M})$ & \multicolumn{5}{l}{ Phospholipid composition $^{\mathrm{a}}$} \\
\cline { 2 - 7 } & PE & DPG & PG & PGL & $\mathrm{X}_{1}$ & $\mathrm{X}_{2}$ & $\mathrm{X}_{3}$ \\
\hline- & $58.8 \pm 3.57$ & $26.1 \pm 1.29$ & $5.6 \pm 0.24$ & $6.1 \pm 0.48$ & $1.2 \pm 0.24$ & $2.2 \pm 0.18$ & - \\
1 & $52.1 \pm 2.42$ & $26.4 \pm 0.79$ & $4.9 \pm 0.24$ & $9.9 \pm 0.91$ & $1.7 \pm 0.06$ & $2.5 \pm 0.04^{*}$ & $2.5 \pm 0.04$ \\
2 & $45.1 \pm 0.54$ & $26.0 \pm 0.73$ & $4.6 \pm 0.06^{*}$ & $16.0 \pm 1.99$ & $1.9 \pm 0.09$ & $3.8 \pm 0.15$ & $2.6 \pm 0.06$ \\
\hline
\end{tabular}

Values of phospholipid contents for control cultures and cultures grown in the presence of $1 \mu \mathrm{M}$ and $2 \mu \mathrm{M}$ AMD were compared by Student's $t$-test. Asterisks indicate significant differences from control values $\left({ }^{*} P<0.05\right)$.

${ }^{a}$ Values are given as $\mathrm{mol} \%$ of total phosphorus content \pm S.D. of three independent determinations. 
in the presence of AMD alter the proportions of the phospholipid classes and contain in their membranes a third unidentified phospholipid $\left(\mathrm{X}_{3}\right), \alpha$-naphtholand ninhydrin-negative (as $\mathrm{X}_{1}$ and $\mathrm{X}_{2}$ ).

AMD, $1 \mu \mathrm{M}$, affects the contents of PE, PG and PGL. Relative to the control, PE decreases by $11.4 \%$ and PG by $12.5 \%$, whereas PGL increases by $62 \%$. The relative increase in PGL reaches $162 \%$ in cultures growing in the presence of $2 \mu \mathrm{M}$ AMD, whereas $\mathrm{PE}$ decreases by $23.3 \%$ and $\mathrm{PG}$ by $17.9 \%$. Only cardiolipin (DPG) remains constant, not being affected by AMD in the growth medium. Therefore, the most significant changes induced by AMD in bacterial phospholipid classes are the decrease of PE and PG and the parallel increase of PGL (Table 1). A similar increase in PGL has been observed when the growth temperature of $B$. stearothermophilus is raised from 48 to $68^{\circ} \mathrm{C}$ [42]. The sugar-containing lipids increase the hydrogen bonding capacity of the bilayer surface. The hydrogen bonding between the hydroxyls of the sugar rings and the surrounding water or the phospholipid neighbors [44,45] takes place and certainly contributes to structure stabilization of the membrane, minimizing the thermal disordering induced by high temperatures. Also, the anticancer drug tamoxifen and the insecticide DDT, which incorporate into the membranes of $B$. stearothermophilus [46,47] affecting the physical state of bacterial lipids [20,46], significantly increase the PGL level $[21,28]$. Apparently, the glycolipid enrichment provides the cell membrane with an increased stability against the perturbations induced by temperature and lipophilic compounds which incorporate into the bilayer disturbing the physical organization. PE is prone to formation of nonlamellar structures like the reversed hexagonal phase [48]. The hexagonal phase propensity of a few membrane lipids is a localized property that relates to the fusion capacity of the membrane and regulates the activity of a variety of enzymes [44]. Under a variety of environmental circumstances, cells carefully balance the contents of lamellar phase-forming and hexagonal phase-forming lipids in membranes. By appropriate metabolic adjustments, membrane lipids are modulated to counteract environmental deleterious effects, in the sense that they preserve the lamellar liquid crystalline phase essential to membrane stability. Therefore, the decrease in the proportions of PE in-

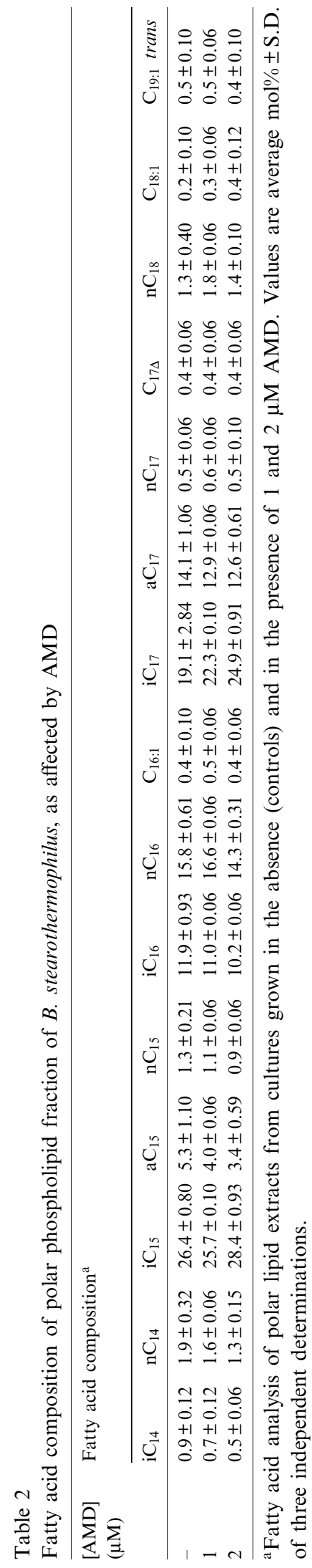


duced by AMD may occur as an adaptation to counteract perturbations of AMD putatively related with disturbances of the lamellar phase. Because electrostatic and hydrophobic forces should determine membrane interactions in the fluid phase [16-18], the presence of AMD is expected to largely affect the equilibrium between the electrostatic interactions of the polar heads and the hydrophobic interactions of the acyl chains of phospholipids. Consequently, in addition to the above considerations, the change in the proportions of the phospholipids is likely to represent a cell adjustment in order to maintain the interactive and repulsive properties at the membrane surface. These alterations also contribute to the reorganization of biological water, i.e., water hydrogen bonded to organic molecules [49].

The acyl chain composition of B. stearothermophilus polar lipids is represented in Table 2. The dominant fatty acid is 13-methyltetradecanoic $\left(\mathrm{iC}_{15}\right.$, $26.4 \%)$ followed by 15-methylhexadecanoic $\left(\mathrm{iC}_{17}\right.$, $19.1 \%)$, palmitic $\left(\mathrm{nC}_{16}, 15.8 \%\right)$, 14-methylhexadecanoic $\left(\mathrm{aC}_{17}, 14.1 \%\right)$, 14-methylpentadecanoic $\left(\mathrm{iC}_{16}\right.$, $11.9 \%)$, 12-methyltetradecanoic $\left(\mathrm{aC}_{15}, 5.3 \%\right)$ and myristic $\left(\mathrm{nC}_{14}, 1.9 \%\right)$. Branched chains, iso- and anteiso-fatty acids, are the predominant components of the polar lipids.

Regarding the fatty acid composition, $1 \mu \mathrm{M}$ AMD has limited effects, as compared with $2 \mu \mathrm{M}$, which alters the proportions of the aliphatic chains significantly. The isomers $\mathrm{iC}_{15}$ and $\mathrm{iC}_{17}$ increase by $7.6 \%$ and $36.4 \%$, respectively. On the other hand, $\mathrm{nC}_{16}$, $\mathrm{aC}_{17}, \mathrm{iC}_{16}, \mathrm{aC}_{15}$ and $\mathrm{nC}_{14}$ decrease by $9.5,10.6$, $14.3,35.8$ and $31.6 \%$, respectively. Branched chains, iso- and anteiso-fatty acids are the predominant aliphatic components of the polar lipids and their relative proportions reach 78\% (Table 3). The branched iso-class is a major group of all isolates and its rel- ative proportion is about $58 \%$ of the total fatty acids. A relative increase $(10 \%)$ of the iso-acids is observed in cell membranes adapted to $2 \mu \mathrm{M}$ AMD, whereas a concomitant decrease of the anteiso-acids (by ca. $22 \%$ ) and straight-chain saturated fatty acids (by $12 \%$ ) is observed. Consequently, the ratio straightchain/branched iso-chain fatty acids decreases from 0.38 (control cultures) to 0.30 (cultures adapted to $2 \mu \mathrm{M}$ AMD). Since iso-branched chains are more loosely packed than straight chains [50], the enrichment of adapted membranes in iso-branched chains compensates for the ordering effect promoted by AMD in the fluid state of polar lipids, when added directly to the lipid dispersions (Fig. 3). The decrease in PE may also contribute to counteracting the ordering effect of AMD, since the propensity to intermolecular hydrogen bonding between PE molecules, with a concomitant increase in order [44], is weakened.

The overall changes in lipid composition may also have the purpose of regulating local bilayer thickness, and thereby preventing unwanted consequences of hydrophobic mismatch in biological membranes [51].

\subsection{Biophysical studies}

Do the changes in lipid composition modulated by the presence of AMD contribute to maintaining lipid packing (membrane order)? To answer this question lipid packing and organization were assessed in polar lipid dispersions of adapted cells by fluorescence polarization and by DSC. Fluorescence polarization of DPH, a probe buried in the bilayer core [37], and of DPH-PA, a probe anchored in the bilayer surface by its charged propionic group [39], was used to determine the modifications in lipid packing across the

Table 3

Fatty acid categories, as affected by AMD

\begin{tabular}{lllll}
\hline$[\mathrm{AMD}](\mu \mathrm{M})$ & \multicolumn{5}{l}{ Fatty acid composition $^{\mathrm{a}}$} & & Total anteiso-acids \\
\cline { 2 - 5 } & Total straight & Total branched & Total iso-acids & $20.5 \pm 2.13$ \\
\hline- & $22.2 \pm 0.85$ & $78.1 \pm 1.08$ & $58.4 \pm 1.04$ & $16.9 \pm 0.00$ \\
1 & $23.0 \pm 0.10$ & $77.0 \pm 0.10$ & $59.7 \pm 0.10$ & $16.0 \pm 0.10$ \\
\hline
\end{tabular}

As in Table 1, values were calculated by Student's $t$-test of paired comparisons, namely, $0 \mu \mathrm{M}$ AMD/1 $\mu \mathrm{M}$ AMD and $0 \mu \mathrm{M}$ AMD/2 $\mu \mathrm{M}$ AMD $(* P<0.05 ; * * P<0.005)$.

${ }^{\text {a }}$ The values are the sums of $\mathrm{mol} \%$ of fatty acids represented in Table 2, arranged in chain categories. 
bilayer thickness. The rotational motions of the probes that result in depolarization of fluorescence are tightly coupled to acyl chain orientational fluctuations [52] and, consequently, reflect the degree of molecular packing (order) in the membrane. DSC provides valuable information on the phase transi-
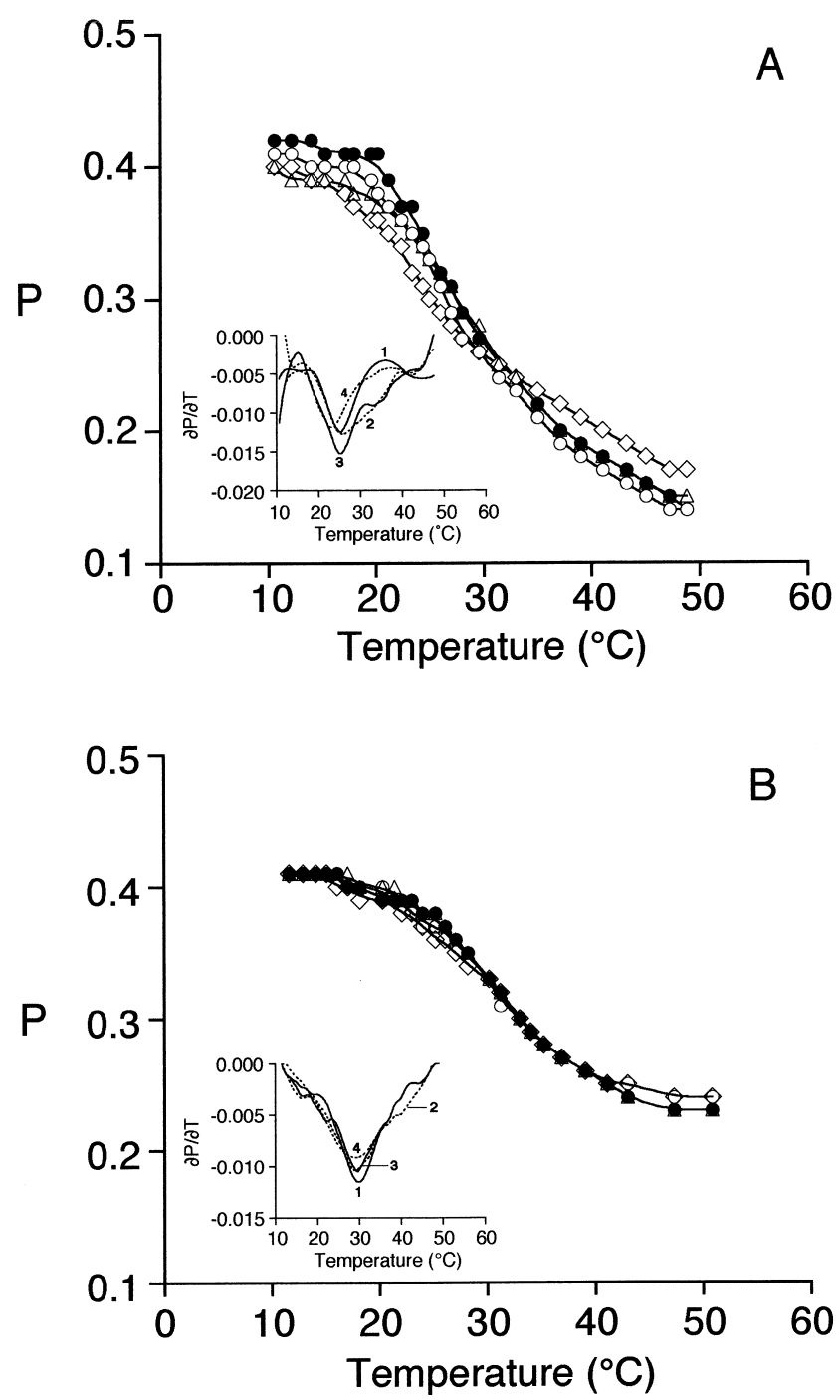

Fig. 3. Fluorescence polarization $(P)$ of DPH (A) and DPH-PA (B), in polar lipid dispersions of cultures grown at $65^{\circ} \mathrm{C}$ in the absence of amiodarone (control cultures, $\mathbf{0})$ and in the presence of $1 \mu \mathrm{M}(\Delta)$ and $2 \mu \mathrm{M}(\bigcirc)$ of the drug (adapted cultures). Also represented is the thermotropic profile, resulting from $40 \mu \mathrm{M}$ amiodarone $(\diamond)$ added directly to polar lipid dispersions of control cultures, which was obtained from a previous work [29]. The first derivative curves 1, 2, 3 and 4, correspond to the main curves with the symbols $\bullet, \Delta, \bigcirc$, and $\diamond$, respectively. As in Fig. 2, standard deviations of the experimental points were too small to be included.

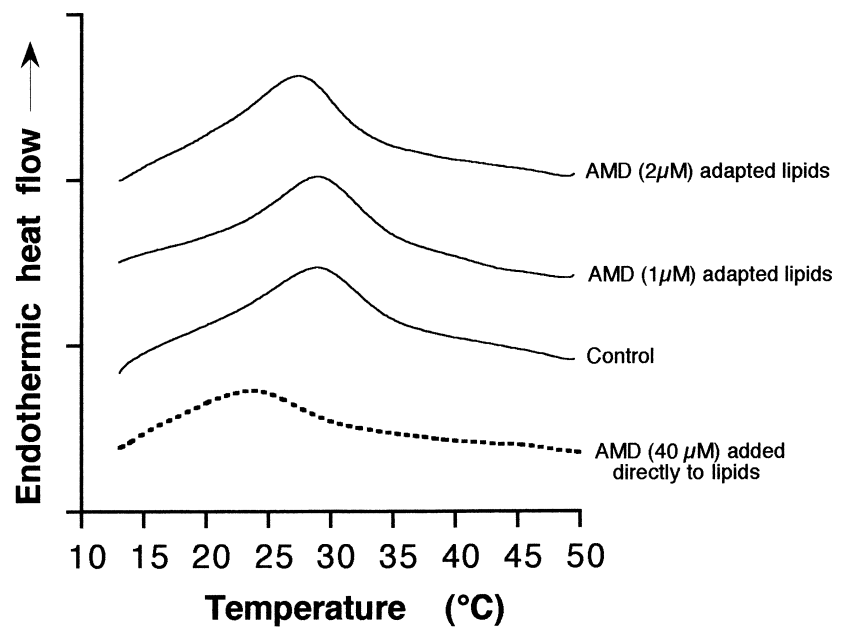

Fig. 4. Thermotropic profiles, obtained by DSC, of polar lipid dispersions of $B$. stearothermophilus grown in the absence of amiodarone (control cultures) or in the presence of 1 and $2 \mu \mathrm{M}$ of the drug (adapted cultures). A DSC profile was also obtained by adding $40 \mu \mathrm{M}$ AMD to polar lipid dispersions from control cultures. The profiles correspond to heating curves. $T_{\mathrm{m}}$ for control cultures and cultures grown in the presence of $1 \mu \mathrm{M}$ and $2 \mu \mathrm{M}$ AMD are $28.8 \pm 0.20,28.9 \pm 0.20$ and $27.4 \pm 0.15$, respectively. A $T_{\mathrm{m}}$ of $23.4 \pm 0.40$ was obtained when AMD $(40 \mu \mathrm{M})$ was added directly to polar lipid dispersions of control cultures. $T_{\mathrm{m}}$ values are means \pm S.D. of three independent experiments. Significance was calculated by Student's $t$-test and was observed for the following paired observations: control cultures/2 $\mu \mathrm{M}$ AMD-adapted cultures and control cultures/40 $\mu \mathrm{M}$ AMD added directly to lipid dispersions.

tion as well as on the enthalpy of melting of molecular membrane components. Also, lateral membrane heterogeneities can be reflected by DSC. Both techniques provide information about the biophysical modifications resulting from lipid composition changes, when AMD is added to the bacterial growth medium.

Fluorescence polarization of DPH and DPH-PA embedded in bacterial polar lipid dispersions from cells grown in the absence of AMD (control cultures) or in the presence of AMD (adapted cultures) is displayed in Fig. 3. The same figure also displays the results obtained when $40 \mu \mathrm{M}$ AMD is added directly to polar lipid dispersions. According to previous studies [42], the polar lipid extracts of control cultures exhibit a broad thermotropic phase transition, with a midpoint $\left(T_{\mathrm{m}}\right)$ at about $28^{\circ} \mathrm{C}$, detected by fluorescence polarization (Fig. 3A,B, solid symbols) and by calorimetry (Fig. 4).

The thermograms obtained by fluorescence polar- 
ization in polar lipid dispersions from cells grown in the presence of 1 and $2 \mu \mathrm{M}$ AMD show some differences relative to controls (without AMD). At temperatures below the phase transition midpoint, the dispersions obtained from adapted cultures show a higher fluidity in the hydrophobic core (Fig. 3A) but not in the outer regions of the bilayer (Fig. 3B), when compared with dispersions from control cultures. As documented in the insets of the figures, it is difficult to detect any alteration in the phase transition midpoint $\left(T_{\mathrm{m}}\right)$ in lipid dispersions of cultures adapted to 1 and $2 \mu \mathrm{M}$ AMD. However, an apparent shifting of $T_{\mathrm{m}}$ (by about $1^{\circ} \mathrm{C}$ ) to lower temperature, statistically significant $(P<0.05)$, is observed by DSC (Fig. 4), with an increase of $\Delta H$ from $29.0 \pm 0.96 \mathrm{~kJ} \mathrm{~mol}^{-1}$ in control cultures, to $34.2 \pm 0.81 \mathrm{~kJ} \mathrm{~mol}^{-1}$ for cells adapted to $2 \mu \mathrm{M}$ AMD. Also, an increase of $\Delta H$ to $34.9 \pm 1.46 \mathrm{~kJ}$ $\mathrm{mol}^{-1}$ is observed for the chain melting phase transition of cells adapted to $1 \mu \mathrm{M}$ AMD, but no shifting in $T_{\mathrm{m}}$ is observed. Fig. 4 also shows that a direct interaction of AMD with polar lipid dispersions induces a decrease in $T_{\mathrm{m}}$ (by $3.2^{\circ} \mathrm{C}$ ), which is statistically significant $(P<0.05)$. Also, a decrease in $\Delta H$ to $19.9 \pm 1.44 \mathrm{~kJ} \mathrm{~mol}^{-1}$, which reflects decreased van der Waals interactions, indicates that lipid chain packing is different from that of adapted cells.

From Fig. 3 it is apparent that, above the phase transition, i.e., in the fluid phase of polar lipid dispersions of cells adapted to $2 \mu \mathrm{M}$ AMD, DPH located in the very fluid bilayer core [53] reflects some disordering, but DPH-PA, displaced to less fluid outer regions of the bilayer [53], reflects limited ordering effects probably assigned to packing density (Fig. 3A,B, open circles).

The overall results, summarized in Figs. 3 and 4, indicate that the thermotropic profiles of polar lipids from adapted cells are more similar to controls, as compared with those obtained when AMD is added directly to polar lipid dispersions. Therefore, the biophysical effects of AMD are partially compensated by growing cells and a bilayer with a general fluidity similar to that of control cultures is obtained.

The above scenarios of adaptive alterations reflect a strong interaction between AMD and membrane lipids. These clues challenge future studies to identify the elements driving the membrane-specific AMD re- sponse, among the components of heterogeneous biomembranes.

\section{Acknowledgements}

This work was supported by Grants Praxis 2/2.1/ $\mathrm{BIO} / 1156 / 94$ and 2/2.1/SAU/1400/95.

\section{References}

[1] E.M. Vaughan-Williams, Adv. Drug Res. 9 (1974) 69-101.

[2] J. Gill, R.C. Heel, A. Fitton, Drugs 43 (1992) 69-110.

[3] G.L. Jendrasiak, T.J. McIntosh, A. Ribeiro, R.S. Porter, Biochim. Biophys. Acta 1024 (1990) 19-31.

[4] M. Trumbore, D.W. Chester, J. Moring, D. Rhodes, L.G. Herbette, Biophys. J. 54 (1988) 535-543.

[5] P. Chatelain, R. Laruel, M. Gillard, Biochem. Biophys. Res. Commun. 129 (1985) 148-154.

[6] P. Chatelain, R. Laruel, P. Vic, R. Brotelle, Biochem. Pharmacol. 38 (1989) 1231-1239.

[7] M.H.C.P.M. Guerreiro, L.C.V. Lopes, A.P. Campello, M.L.W. Klüppel, Arq. Biol. Tecnol. 29 (1986) 621-631.

[8] J.W. Card, B.R. Lalonde, E. Rafeiro, A.S. Tam, W.J. Racz, J.F. Brien, T.M. Bray, T.E. Massey, Toxicol. Lett. 98 (1998) 41-50.

[9] B. Fromenty, C. Fisch, A. Berson, P. Letteron, D. Larrey, D. Pessayre, J. Pharmacol. Exp. Ther. 255 (1990) 1377-1384.

[10] D.F. Gray, A.S. Mihailidou, P.S. Hansen, K.A. Buhagiar, N.L. Bewick, H.H. Rasmussen, D.W. Whalley, J. Pharmacol. Exp. Ther. 284 (1998) 75-82.

[11] D. Moreau, F. Clauw, L. Martine, A. Grynberg, L. Rochette, L. Demaison, Mol. Cell. Biochem. 194 (1999) 291-300.

[12] J. Sikkema, J.A.M. de Bont, B. Poolman, Microbiol. Rev. 59 (1995) 201-222.

[13] E.E. Williams, Am. Zool. 38 (1998) 280-290.

[14] P. Chatelain, J. Ferreira, R. Laruel, J.M. Ruysschaert, Biochem. Pharmacol. 35 (1986) 3007-3013.

[15] P. Chatelain, R. Brotelle, R. Laruel, Biochem. Pharmacol. 36 (1987) 1564-1565.

[16] A.-M. Sautereau, C. Tournaire, M. Suares, J.-F. Tocanne, N. Paillous, Biochem. Pharmacol. 43 (1992) 2559-2566.

[17] M.C. Antunes-Madeira, R.A. Videira, M.L.W. Klüppel, V.M.C. Madeira, Int. J. Cardiol. 48 (1995) 211-218.

[18] Y. Attal, X.A. Cao, G. Perret, E. Taillandier, Chem. Pharm. Bull. 45 (1997) 1317-1322.

[19] M.T. Silva, J.C.F. Sousa, J.J. Polónia, P.M. Macedo, J. Bacteriol. 137 (1979) 461-468.

[20] M.M. Donato, A.S. Jurado, M.C. Antunes-Madeira, V.M.C. Madeira, Arch. Environ. Contam. Toxicol. 33 (1997) 109-116.

[21] C. Luxo, A.S. Jurado, V.M.C. Madeira, Biochim. Biophys. Acta 1369 (1998) 71-84. 
[22] H.R. Kaback, Biochim. Biophys. Acta 265 (1972) 367-416.

[23] I.R. Booth, Microbiol. Rev. 49 (1985) 359-378.

[24] J.B. Stock, A.M. Stock, J.M. Mottonen, Nature 344 (1990) 395-400.

[25] B.L. Trumpower, R.B. Gennis, Annu. Rev. Biochem. 63 (1994) 675-716.

[26] N.J. Russel, Trends Biochem. Sci. 9 (1984) 108-112.

[27] A.S. Jurado, A.C. Santana, M.S. da Costa, V.M.C. Madeira, J. Gen. Microbiol. 133 (1987) 507-513.

[28] M.M. Donato, A.S. Jurado, M.C. Antunes-Madeira, V.M.C. Madeira, Arch. Environ. Contam. Toxicol. 33 (1997) 341-349.

[29] S.M.L.J. Rosa, M.C. Antunes-Madeira, A.S. Jurado, V.M.C. Madeira, Appl. Biochem. Biotech. (2000) (in press).

[30] E.G. Bligh, W.J. Dyer, Can. J. Biochem. Physiol. 37 (1959) 911-917.

[31] G.R. Bartlett, J. Biol. Chem. 234 (1959) 466-468.

[32] C.J.F. Böttcher, C.M. van Gent, C. Pries, Anal. Chim. Acta 24 (1961) 203-204.

[33] K. Randerath, Thin-Layer Chromatography, 2nd edn., pp. 151-154, Academic Press, London, 1968.

[34] J.C. Dittmer, R.L. Lester, J. Lipid Res. 5 (1964) 126-127.

[35] H. Jacin, A.R. Mishkin, J. Chromatogr. 18 (1965) 170-173.

[36] D.F. Silbert, R.C. Ladenson, J.L. Honegger, Biochim. Biophys. Acta 311 (1973) 349-361.

[37] M. Shinitzky, Y. Barenholz, Biochim. Biophys. Acta 515 (1978) 367-394.

[38] B.J. Litman, Y. Barenholz, Methods Enzymol. 81 (1982) 678-685.
[39] P.J. Trotter, J. Storch, Biochim. Biophys. Acta 982 (1989) 131-139.

[40] P.W.M. van Dijck, B. de Kruijff, L.L.M. van Deenen, J. de Gier, R.A. Demel, Biochim. Biophys. Acta 455 (1976) 576587.

[41] R.D. Koynova, A.I. Boyanov, B.G. Tenchov, FEBS Lett. 187 (1985) 65-68.

[42] A.S. Jurado, T.J.T. Pinheiro, V.M.C. Madeira, Arch. Biochem. Biophys. 289 (1991) 167-179.

[43] R.L. Biltonen, D. Lichtenberg, Chem. Phys. Lipids 64 (1993) 129-142.

[44] J.M. Boggs, Biochim. Biophys. Acta 906 (1987) 353-404.

[45] T. Gutberlet, J. Frank, H. Bradaczek, W. Fisher, J. Bacteriol. 179 (1997) 2879-2883.

[46] C. Luxo, A.S. Jurado, J.B.A. Custódio, V.M.C. Madeira, Toxicol. In Vitro 10 (1996) 463-471.

[47] M.M. Donato, M.C. Antunes-Madeira, A.S. Jurado, V.M.C. Madeira, Bull. Environ. Contam. Toxicol. 59 (1997) 696-701.

[48] P.R. Cullis, B. de Kruijff, Biochim. Biophys. Acta 559 (1979) 399-420.

[49] W.R. Klemm, Alcohol 15 (1998) 249-267.

[50] D.K. Rice, D.A. Cadenhead, R.N.A.H. Lewis, R.N. McElhaney, Biochemistry 26 (1987) 3205-3210.

[51] J.A. Killian, Biochim. Biophys. Acta 1376 (1998) 401-416.

[52] B.R. Lentz, Chem. Phys. Lipids 50 (1989) 171-190.

[53] W. Chefurka, R.C. Chatelier, W.H. Sawyer, Biochim. Biophys. Acta 896 (1987) 181-186. 